\title{
AIAA 2002-0841 \\ Summary of Data from the \\ First AIAA CFD \\ Drag Prediction Workshop
}

David W. Levy, Tom Zickuhr

Cessna Aircraft Co.,Wichita, KS

John Vassberg, Shreekant Agrawal

The Boeing Co., Long Beach, CA

Richard A. Wahls, Shahyar Pirzadeh, Michael J. Hemsch

NASA Langley Research Center, Hampton, VA

\section{$40^{\text {th }}$ AIAA Aerospace Sciences Meeting January 14-17, 2002, Reno, NV}




\section{AIAA-2002-0841}

\section{Summary of Data from the First AIAA CFD Drag Prediction Workshop}

\author{
David W. Levy ${ }^{*}$, Tom Zickuhr ${ }^{\dagger}$ \\ Cessna Aircraft Co., Wichita, KS
}

\author{
John Vassberg ${ }^{\ddagger}$, Shreekant Agrawal $^{\ddagger}$ \\ The Boeing Co., Long Beach, CA
}

Richard A. Wahls ${ }^{\S}$, Shahyar Pirzadeh ${ }^{\llbracket}$, Michael J. Hemsch ${ }^{\#}$
NASA Langley Research Center, Hampton, VA

\begin{abstract}
The results from the first AIAA CFD Drag Prediction Workshop are summarized. The workshop was designed specifically to assess the state-of-the-art of computational fluid dynamics methods for force and moment prediction. An impartial forum was provided to evaluate the effectiveness of existing computer codes and modeling techniques, and to identify areas needing additional research and development.

The subject of the study was the DLR-F4 wing-body configuration, which is representative of transport aircraft designed for transonic flight. Specific test cases were required so that valid comparisons could be made. Optional test cases included constant- $\mathrm{C}_{\mathrm{L}}$ drag-rise predictions typically used in airplane design by industry. Results are compared to experimental data from three wind tunnel tests.

A total of 18 international participants using 14 different codes submitted data to the workshop. No particular grid type or turbulence model was more accurate, when compared to each other, or to wind tunnel data. Most of the results overpredicted $\mathrm{C}_{\mathrm{Lo}}$ and $\mathrm{C}_{\mathrm{Do}}$, but induced drag $\left(\mathrm{dC}_{\mathrm{D}} / \mathrm{dC}_{\mathrm{L}}{ }^{2}\right)$ agreed fairly well. Drag rise at high Mach number was underpredicted, however, especially at high $\mathrm{C}_{\mathrm{L}}$. On average, the drag data were fairly accurate, but the scatter was greater than desired. The results show that well-validated Reynolds-Averaged Navier-Stokes CFD methods are sufficiently accurate to make design decisions based on predicted drag.
\end{abstract}

\footnotetext{
* Engineer Specialist, Senior Member AIAA

$\uparrow$ Engineer Specialist, Member AIAA

* Boeing Technical Fellow, AIAA Associate Fellow

$\S$ Asst. Head, Configuration Aerodynamics Branch, AIAA Associate Fellow

` Senior Research Engineer

\# Aerospace Engineer, AIAA Associate Fellow

Copyright $(2002$ by David W. Levy. Published by the American Institute of Aeronautics and Astronautics, Inc. with permission.
}

Introduction

It is well known that CFD is widely used in the aircraft industry to analyze aerodynamic characteristics during conceptual and preliminary design. All major airframe manufacturers world-wide now have the capability to model complex airplane configurations using CFD methods. To be useful as a design tool, the accuracy of a method must be determined through some kind of verification and validation process. As CFD methods have evolved, many such studies have been conducted. Some are reported in the open literature, but, due to deficiencies in the published studies, many more are conducted in-house with proprietary data that cannot be disseminated freely throughout the industry.

The majority of published studies describe CFD algorithm development. It is common to see results on relatively simple configurations without any comparison to experimental data. This is even true of literature that does concentrate on drag prediction. When comparisons are made, usually they are pressure distributions. To be sure, accurate pressure prediction is important, and many CFD groups make early configuration decisions based on evaluation of pressures alone. But pressure is not the whole story.

CFD methods and computer capabilities have advanced remarkably in the past decade. It is now practical to routinely model complete airplane configurations and analyze multiple flight conditions using Reynoldsaveraged Navier-Stokes (RANS) methods. With this capability, the focus of the analysis has shifted from detailed examination of a single solution to trends with angle of attack and Mach number.

Because of this shift in focus, we must now verify the accuracy of integrated forces and moments. In particular, the ability to predict drag accurately is important. Once this ability is demonstrated (to the nonCFD community), the credibility of CFD will improve dramatically. 
AIAA-2002-0841

As mentioned above, published validation studies are not very common, although they do exist ${ }^{1-5}$. This is especially true of configurations with experimental data that are in the public domain. Perhaps the closest type of study to the present work is described in Reference 6. This work was published in 1997.

It is in this context that the present workshop was conceived. A technical working group was formed within the AIAA Applied Aerodynamics Technical Committee in 1998 with a focus on CFD drag and transition prediction. This group was composed primarily of members from industry, and the consensus was that, while CFD was beginning to be used in industry for drag prediction, it was unclear what the state of the art was. It was decided to conduct an international workshop inviting participants from universities, research labs, and industry. Several members of the technical working group formed an organizing committee to plan and conduct the workshop.

The goal of the workshop was to assess the state-of-theart of CFD with a primary focus on CFD drag prediction. By bringing together a large sampling of experts in this field, who were willing to share their experiences in the pursuit of this critical and elusive quantity, the state-of-the-art may even be advanced. Several key features of the workshop were designed to facilitate this end:

1. The subject geometry, the DLR-F4 wing-body ${ }^{7}$, was chosen as simple enough to do high quality computations and still relevant to the type of configuration useful to industry. A large body of experimental data is also available in the public domain for this configuration.

2. Several test cases were chosen ranging from a single $\mathrm{Mach} / \mathrm{C}_{\mathrm{L}}$ condition, which is within reach of most CFD groups, to a constant $C_{L}$ Mach sweep typically used by industry to determine drag-rise characteristics

3. A standard set of grids was provided to the participants to reduce the variability in the results. All participants were required to submit results for the single $\mathrm{Mach} / \mathrm{C}_{\mathrm{L}}$ case on one of the standard grids. Participants were also encouraged to generate their own grids using techniques and standards developed from their experience.

4. A rigorous statistical analysis was performed on these results to establish confidence levels in the data.
The geometry, test cases, and grids all combine to encourage wide participation and test the state-of-the-art in the context of engineering application.

In the following pages, a complete description of the geometry is included. This includes how it was processed from a series of point-defined stations to a completely surfaced, loft definition suitable for grid generation. The standard grids are described for the multiblock structured, unstructured, and overset options. Then the test cases are defined.

An overview of the participation is presented. The results are summarized, including lift curves, drag polars, pitching moment, and drag rise characteristics. The drag data are also broken out by grid type, turbulence model, and code to identify trends with these parameters. Complete listings of the data, including presentations by the participants, are included in the workshop proceedings ${ }^{8}$.

\section{Geometry Description}

In choosing the geometry to be used for the workshop, several criteria were considered. First, the geometry needed to be relatively simple, so that participation in the workshop would be encouraged. However, the geometry also needed to be complex enough to test users' capabilities and to be relevant to the type of work done in industry. These two factors led to the choice of a wing-body as a good compromise.

It was also desired to have experimental data available with which to make comparisons. The subject of the test needed to be available and well defined. It was beyond the resources of the organizing committee to design a new, on-purpose geometry and to conduct the required testing.

A few options that fit these requirements were known to the organizing committee, but the one that had the largest body of data available was the DLR-F4 wingbody, shown in Figure 1. The geometry and experimental data are described in detail in AGARD report 303 (Ref. 7), which is a document specifically designed to provide data for CFD verification and validation.

The geometry of the body for the DLR-F4 is defined in Ref. 7 with 90 defining stations composed of 66 points each. The wing is defined with 4 stations of 145 points each. These coordinates were uploaded into CATIA, and surfaces fit to the data using standard lofting techniques. Certain features, such as the windshield and horizontal tail flat were not explicitly defined, but were 
AIAA-2002-0841

easily gleaned from the data. The nose tip, tail cap, and wing tip were added as defined in AGARD 303. Also note that the lower surface of the wing required a slight extrapolation to intersect with the body, and that there is no wing-body fairing. As a final modification, the wing was twisted by approximately $0.4^{\circ}$, per AGARD 303, to simulate a loaded condition.

A large amount of experimental data is also included with AGARD 303. The same model was tested in three different wind tunnels. The bulk of the data concentrates on wing pressure profiles. Pressures are given for a constant $\mathrm{C}_{\mathrm{L}}=0.5$ Mach sweep from $\mathrm{M}_{\infty}=0.60$ to 0.82 , and for $\mathrm{C}_{\mathrm{L}}=0.30,0.40,0.50$, and 0.60 at $\mathrm{M}_{\infty}=0.75$. Force and moment data are supplied for three alpha sweeps, at $\mathrm{M}_{\infty}=0.60,0.75$, and 0.80 . All data are for a Reynolds number of $3 \times 10^{6}$ based on the wing mean geometric chord, and boundary layer transition is fixed according to a defined trip strip pattern. Standard methods for correcting the data due to wall, buoyancy, and sting effects were used by each tunnel, however, the correction methods were not uniform. An unfortunate shortcoming of the data is that the drag coefficient values are only given to a precision of three decimals ( \pm 0.001 , or 10 counts). Attempts to obtain more precise data were unsuccessful.

\section{Standard Grids}

To minimize variation in the results and facilitate the statistical analysis, a set of standard grids were generated. These grids were built to a consistent set of specifications regarding spacing and distribution. In this way, variations simply due to gridding differences could be held to a minimum. The participants were required to submit the results from the first test case using one of the required grids. A sampling of the grid specifications are listed in Table 1.

\section{Table 1. Grid specifications used to generate the Standard Grids.}

Wing LE Spacing:

Wing TE spacing:

Spanwise Spacing at Wing Tip:

Cells on Blunt TE:

First BL Cell Normal Spacing:

BL Cell Stretching Ratio:

Far Field Boundary Distance:

$0.1 \% \mathrm{MAC}$
$0.125 \% \mathrm{MAC}$
$0.5 \%$ span
4
$.001 \mathrm{~mm}$
1.2 to 1.25
50 chords

a substantial effort. Several of the participants would not have been able to do the work if they had been required to generate their own grids. However, the participants were encouraged to generate grids using best practices they had learned through experience. By sharing the details of their gridding techniques, the state-of-the-art can perhaps be improved.

Four grids were built for use with the following types of codes:

1. Multiblock Structured

2. Unstructured, cell-based

3. Unstructured, node-based

4. Overset

The multiblock structured grid was built using the ICEM CFD module Hexa. It has 49 blocks all with one-to-one point matching at the block boundaries, and up to three levels of multigrid are available. Blocks around the wing and body used an O-grid topology.

The two unstructured grids were built with VGRIDns. They both used the same relative distribution, but global refinement was used for the nodal grid to get sufficient resolution for node-based codes. The grids were fully tetrahedral. However, an advancing layer technique was used for the boundary layer grids, so the structure was present to reconstruct prisms in the boundary layer.

The surface mesh for the overset grid was built with Gridgen V13. The surface abutting volume grids were generated with HYPGEN. Intermediate fields were captured with box grids, and finally a far-field box grid surrounded the entire geometry and went out to the outer boundary. Hole cutting and fringe point coupling was performed with GMAN.

A summary of the grid statistics for the standard grids are listed in Table 2.

Table 2. Grid Statistics for the Standard Grids.

\begin{tabular}{|l|r|l|c|c|}
\hline Grid & Nodes & Cells & $\begin{array}{l}\text { Bndry } \\
\text { Nodes }\end{array}$ & $\begin{array}{l}\text { Bndry } \\
\text { Faces }\end{array}$ \\
\hline $\begin{array}{l}\text { Structured } \\
\text { Multiblock }\end{array}$ & $3,257,897$ & $3,180,800$ & --- & 153,376 \\
\hline $\begin{array}{l}\text { Unstructured } \\
\text { Cell Based }\end{array}$ & 470,427 & $2,743,386$ & 23,290 & 46,576 \\
\hline $\begin{array}{l}\text { Unstructured } \\
\text { Node Based }\end{array}$ & $1,647,810$ & $9,686,802$ & 48,339 & 96,674 \\
\hline Overset & $3,231,377^{*}$ & --- & 54,445 & --- \\
\hline
\end{tabular}

*Non-Blanked Nodes

It was recognized that the standard grids could not possibly meet all solvers' requirements and could have shortcomings affecting certain solutions. Participants 
AIAA-2002-0841

were encouraged to generate their own grids according to their best practices and requirements. Also, some participants were unable to use any of the grids due to incompatibility with their codes. In these cases, they submitted data only with their grids.

\section{Test Case Description}

There were several goals that contributed to the selection of the test cases. From the outset, it was desired for this to be a controlled study, so that the variation in the results could be minimized wherever possible, and suitable for a statistical analysis. As with the geometry, the set of test cases needed to be simple enough to maximize participation yet also test the practicality of the CFD codes when used in an industry environment. A set of required cases were determined that would enhance participation:

\section{Required Cases:}

Case 1: $\mathrm{M}_{\infty}=0.75, \mathrm{C}_{\mathrm{L}}=0.500 \pm 0.005$

Case 2: $\mathrm{M}_{\infty}=0.75, \alpha=-3^{\circ},-2^{\circ},-1^{\circ}, 0^{\circ}, 1^{\circ}, 2^{\circ}$

All cases were to be run at the wind tunnel test $\mathrm{R}_{\mathrm{Nc}}=3 \times 10^{6}$ based on the wing mean geometric chord. However, it was specified that the transition pattern specified in Ref 7 was not to be used. Because transition specification for 3D RANS codes is still relatively rare, all cases were run "fully turbulent." Note that this term is still fairly inexact, as different turbulence models will still take some time to build up the turbulence level.

For Case 1, one of the standard grids was to be used if possible. This requirement was designed to enhance the statistical analysis by removing variability due to grids as much as possible. Since the workshop was focused on drag accuracy, a fixed $C_{L}$ was chosen instead of $\alpha$, to remove any variation in $C_{D}$ due to variations in $C_{L}$. For Case 2, the participants were allowed to use their own grids, if desired.

\section{Optional Cases:}

Case 3: $\mathrm{M}_{\infty}=.50, .60, .70, .75, .76, .77, .78, .80$ $\mathrm{C}_{\mathrm{L}}=0.500 \pm 0.005$

Case 4: $\mathrm{M}_{\infty}=.50, .60, .70, .75, .76, .77, .78, .80$ $\mathrm{C}_{\mathrm{L}}=0.400,0.500,0.600 \pm 0.005$

Note that Case 4 includes Case 3. These cases are increasingly more difficult, but are more typical of the type of data needed and used by industry. Of particular interest was whether separation present at higher Mach number $/ \mathrm{C}_{\mathrm{L}}$ combinations would be accurately predicted.

\section{Overview of Methods and Data Submitted}

A total of 18 participants attended the workshop, giving results from 14 different code types. Many participants submitted more than one set of results, exercising different options in their codes (e.g., turbulence models) and/or using different grids. A breakdown of the total submittals for each case is shown below:

\begin{tabular}{|r|c|c|c|c|}
\hline Case & 1 & 2 & 3 & 4 \\
\hline Submittals & 35 & 28 & 10 & 9 \\
\hline
\end{tabular}

For the 18 participants, the breakdown of grid types that were used is shown below:

\begin{tabular}{|c|c|c|c|}
\hline $\begin{array}{c}\text { Multiblock } \\
\text { Structured }\end{array}$ & Unstructured & Overset & Cartesian \\
\hline 8 & 7 & 2 & 1 \\
\hline
\end{tabular}

Of the Case 1 results submitted, 21 used one of the standard grids, and 14 used other grids. A general breakdown of the turbulence models used for the Case 2 results is shown below:

\begin{tabular}{|c|c|c|c|}
\hline $\begin{array}{c}\text { Spalart- } \\
\text { Allmaras }\end{array}$ & $\mathrm{k}-\omega$ & $\mathrm{k}-\varepsilon$ & other \\
\hline 14 & 10 & 2 & 2 \\
\hline
\end{tabular}

A few of the Spalart-Allmaras results specified a particular version of the model, but most did not do so. The k- $\omega$ results include the Wilcox, Menter SST, EASM, and LEA models. Three of the participants used wall-functions.

\section{Results and Discussion}

The first required case was run at a specified $C_{L}$ and Mach number, and one of the standard grids was to be used. Average quantities are listed in Table 3.

Table 3. Summary of Results for Case 1: $M_{\infty}=0.75$, $\mathrm{C}_{\mathrm{L}}=0.500, \mathrm{R}_{\mathrm{Nc}}=3 \times 10^{6}$.

\begin{tabular}{|l|r|r|r|r|}
\hline & \multicolumn{1}{|c|}{ Avg } & \multicolumn{1}{c|}{ Min } & \multicolumn{1}{c|}{ Max } & Expmt* \\
\hline Alpha & -.237 & -1.000 & 1.223 & .177 \\
\hline$C_{L}$ & .5002 & .4980 & .5060 & .500 \\
\hline$C_{D \text { Total }}$ & .03037 & .02257 & .04998 & .02865 \\
\hline$C_{\text {D Pressure }}$ & .01698 & .01211 & .03263 & --- \\
\hline$C_{D \text { Viscous }}$ & .01327 & .00499 & .02576 & --- \\
\hline$C_{M}$ & -.1559 & -.2276 & .0481 & -.1303 \\
\hline
\end{tabular}

*Interpolated from wind tunnel data in Ref 7. 
AIAA-2002-0841

The CFD codes tended to overpredict $\mathrm{C}_{\mathrm{L}}$ at a given alpha. To achieve the target $\mathrm{C}_{\mathrm{L}}$, an average offset of $-0.414^{\circ}$ was required. The CFD results also tended to predict drag to be too high by an average of 17.2 counts, and the $\mathrm{C}_{\mathrm{M}}$ to be off by -0.0256 (nose down).

There are several reasons why the CFD results were not expected to match the experimental data. First, the CFD runs were all specified to be fully turbulent. Since there was no laminar run ahead of the trip strips, as in the experimental data, the drag should be too high. The decrease in drag due to the laminar portion of the boundary layer is estimated to be 13 counts. Taking this into account, the error is approximately 4.2 counts. Also, the CFD runs were all computed in free air, and the sting mount was not modeled. The effects of these differences are difficult to quantify without specific study to identify them.

The validity of these comparisons - free air CFD to wind tunnel data, warrants some discussion. To match wind tunnel data accurately, the computations should include the mounting hardware and tunnel walls (perhaps porous or slotted), and the tunnel data should not include some of the corrections normally applied (e.g. blockage). But this is not usually done in practice, and could not be done here since the uncorrected data were not available. Even though the tunnel data are corrected to a free air condition, the correction process introduces some error. In this respect, the CFD simulations more accurately represent the real case of free air than the wind tunnel. The final conclusion is that neither the CFD nor the experiment are exact. There is a much larger body of experience with wind tunnel testing, so there is wider acceptance of its validity. As more experience is gained with CFD, it too will gain acceptance. The comparisons made in this paper should be interpreted with these thoughts in mind.

It is also seen from Table 3 that there is a considerable amount of scatter in the data. There is a range of over 270 counts in the drag data, which is quite unacceptable. More detailed examination of the data, Shown in Figure 2 , shows that the majority of the results are much better than indicated by the total range. There are five bad results, or "outliers," which can be identified. Some of these outliers were determined to be due to errors in the runs performed by participants. The one Euler/IBL submission also had a larger error than most of the other results, which is not unreasonable.

A comprehensive statistical analysis of the data is performed in Ref. 9. The effects of outliers and a quantitative determination of the confidence level of the results are included.
A typical pressure profile for Case 1 is shown in Figure $3 a$, taken from Ref 8 . It shows the effect of a mismatch in $\alpha$ : the upper surface pressure peak is lower than experiment, and the post-shock Mach number is too high. Most of the participants had similar results. During the discussion associated with the presentations, many hypotheses were offered as to the source of the mismatch, including offsets in angle of attack and a change in the effective Mach number due to blockage. The general attitude changed dramatically when the presentation was made for the code SAUNA, with the "better" result shown in Figure 3b. These results also differed from most of the others in that the lift and pitching moment agreement was very good. These results were not run on the standard grid, as it was not compatible with the code. Also of note, the geometry for this result was altered in that the wing trailing edge and tip were made to be sharp. The point was raised that it may be better to avoid the complication of a blunt trailing edge, especially if the relevant flow features aren't captured anyway. However, this is not the position of the DPW organizing committee. In fact, technical issues such as this one are precisely what the workshop was intended to expose. The first step towards correcting a problem is to recognize that it exists. Furthermore, blunt trailing edges can be an integral element to transonic airfoil design.

Case 2 is representative of a typical alpha-sweep which is performed in wind tunnel testing and can be used to compare trends with angle of attack and lift. The lift curve results for all Case 2 submissions is shown in Figure 4. Note that several of these cases were run on different grids than for Case 1, so there are some differences from the data in Figure 2. As with the Case 1 results, most of the data are consistently higher in $\mathrm{C}_{\mathrm{L}}$ at a given $\alpha$ than the wind tunnel data. The average lift curve slope (derived from linear curve fits), however, is very close to the experimental value. Several of the results show nonlinearities at $\alpha=2^{\circ}$, which agrees with the experiment. The bulk of the CFD data tend to agree with each other, however, four outliers can be identified. No trends with grid type (indicated by the line type) or turbulence model (indicated by the line color) can be readily identified from this graphical analysis.

The drag polars for Case 2 are shown in Figure 5. An increase in the relative scatter is apparent, which might be expected for $C_{D}$. Most of the results are consistently higher than the tunnel data - similar to the Case 1 results. Again, the four outliers are seen. A better appreciation of the induced drag characteristics is gained by plotting $\mathrm{C}_{\mathrm{D}}$ vs. $\mathrm{C}_{\mathrm{L}}{ }^{2}$, shown in Figure 6 , which is a 
AIAA-2002-0841

linear relationship for an ideal drag polar. The average slope is very close to the experiment.

Pitching moment results are shown in Figure 7. Note that this configuration has no tail, and is almost neutrally stable. There is a larger scatter band in these results - in both the CFD and the wind tunnel data. Most of the results are too negative. It should be noted that the one set that matches the wind tunnel data very well (indicated by the symbol "Y" in Figure 7), is from the same results that produced the "better" pressure match in Figure $3 b$. The missed pressure distribution on the wing may contribute to the pitching moment error.

In a further attempt to glean trends in the drag data related to basic method attributes, Figure 8 shows plots of idealized profile drag for each of the major code types submitted: multiblock structured, unstructured, overset, and other (Cartesian-Euler/IBL). Idealized profile $\mathrm{drag}^{2}$ is defined by the formula:

$$
\mathrm{C}_{\mathrm{DP}}=\mathrm{C}_{\mathrm{D}}-\mathrm{C}_{\mathrm{L}}^{2} /(\pi \mathrm{AR})
$$

where $\mathrm{AR}$ is the aspect ratio. Plotting $\mathrm{C}_{\mathrm{DP}}$ generally results in a more compact presentation of the data, allowing more expanded scales. The two methods with the most results (multiblock structured and unstructured) both have considerable scatter, overpredict basic drag levels, and have one or more outliers. The multiblock structured results have a bit more scatter, but represent a larger number of different codes and turbulence models than the unstructured results. Both of the overset results are from the same code and grid, so their agreement is to be expected. For all methods, drag at higher $\mathrm{C}_{\mathrm{L}}$ is underpredicted, which would indicate that drag due to shock-induced separation is not captured. However, for attached flow conditions, the averaged CFD results are about 10-15 counts higher than the test data; this is consistent with the 13-count shift between fully turbulent (CFD) and partially laminar (windtunnel) flows.

Results are sorted by turbulence model type in Figure 9. The most common model used is from Spalart and Allmaras, and again it is seen that code and grid type contribute to the scatter. The Menter SST k- $\omega$ results tend to agree better with experiment at higher $C_{L}$, indicating that the CFL3D implementation does a better job predicting the non-ideal drag than the other turbulence models. Overall, no particular turbulence model appears to be more consistent across code and grid types than the others.

The characteristics of different codes are shown in Figure 10. Here it is comforting to see that a code run on the same grid with the same turbulence model will give the same result regardless of where or by whom it is run.

The discussion of the Case 3 and 4 results is combined. There was only one submission that ran Case 3 but did not complete all of Case 4. Two participants augmented Case 4 with a drag rise curve for $C_{L}=0.30$. Figure 11 shows the drag rise characteristics. Wind tunnel data are only available for $\mathrm{M}_{\infty}=0.60,0.75$, and 0.80 . The general scatter at the lower Mach numbers is similar to the previous data. The knee of the drag rise curves appear to be in the right place, but the CFD results tend to underpredict the drag more at higher $\mathrm{Mach} / \mathrm{C}_{\mathrm{L}}$ combinations. This would indicate that shock induced separation is not accurately predicted.

As the participants presented their results, a lively discussion often ensued that was open and honest. Many of the users had difficulty with the standard multiblock grid, leading to less accurate results. The organizing committee acknowledges that the grid was of lower quality than desired.

Much of the discussion centered on the ability to predict the basic pressure distribution on a wing, and the effects of trailing edge modeling techniques. Some of the participants ran cases at a fixed $\alpha$ to compare with the wind tunnel data. Generally, the suction peak magnitude agreed better but the shock was located too far aft. Many of the participants argued that leading edge grid refinement and boundary layer transition can affect the basic pressure distribution as well. At least one participant pointed out that, to properly simulate the flow, basic freestream turbulence levels and length scales are required. These are parameters that are typically "hard-wired" into codes and are not specified by the user.

Questions regarding details of the experimental data such as wall corrections, blockage corrections, and effects of the sting mounts were raised which, unfortunately, could not be answered. A better understanding of experimental techniques and wind tunnel corrections by the CFD community could lead to more accurate validation of CFD codes.

\section{Conclusions and Recommendations}

A workshop was held with the specific goal to assess the state-of-the-art of computational methods to predict the drag of a transport aircraft wing-body configuration. Standardized grids and test cases were used to facilitate the comparisons. A large body of data was gathered 
AIAA-2002-0841

from 18 international participants and presented in an objective manner.

In general, the CFD lift and minimum drag levels are higher than the wind tunnel results. Non-parabolic drag is slightly lower than experiment at higher Mach number $/ \alpha$ combinations (i.e., post-buffet conditions) where separation is present. While the comparisons with experiment were reasonable, the large amount of scatter does not promote a high level of confidence in the results. However, much of the scatter was due to "outlier" solutions that were generally agreed to be in error. The data shows no clear advantage of any specific grid type or turbulence model.

Using the standard grids did not help to improve the consistency of the results. The multiblock structured grid did not have the desired quality, which degraded the performance of several of the codes.

The overall level of scatter is too high, and needs to be reduced to determine overall accuracy and trends with grid type, turbulence model, etc. Future work should try to identify sources of the scatter (e.g. grid quality).

Although the scatter is larger than desired, much of it is due to the various grids, codes, turbulence models, etc. that were used. A single organization that uses one or two codes and consistent grid generation and modeling techniques, will experience more consistent results. In this sense, CFD is quite useful as an engineering tool to evaluate relative advantages of one configuration over another.

More experience needs to be gained where CFD is used in conjunction with wind tunnel data on development projects that culminate in a flight vehicle. Then the methods can be "calibrated" to a known outcome. Note that experimental methods went through a similar process long ago. Wind tunnel testing is not regarded as "perfect", but it is useful as an engineering tool because its advantages and limitations are well known. CFD needs to go through the same process.

A second workshop is in the very early planning stages, and is tentatively scheduled for the Summer of 2003. Perhaps the most important item to be decided for this workshop is what type of configuration to use. Many participants believe there are basic issues that are not done well yet, and that the configuration should be simpler. Others were ready to proceed to more complicated configurations, such as a wing-bodynacelle, and continue evaluation as an engineering tool.

\section{Acknowledgements}

The authors wish to thank the AIAA Technical Activities Committee and the AIAA Applied Aerodynamics Technical Committee for financial support of the workshop. We also wish to thank our respective companies for their support. Finally, thanks go to the participants, for without their contributions this workshop would not have been possible.

\section{References}

1. Tinoco, E.N., "The Changing Role of Computational Fluid Dynamics in Aircraft Development," AIAA98-2512, 1998.

2. Tinoco, E.N., "An Assessment of CFD Prediction of Drag and Other Longitudinal Characteristics," AIAA 2001-1002, 2001.

3. Agrawal, S. and Narducci, R., "Drag Prediction using Nonlinear Computational Methods," AIAA 2000-0382, 2000.

4. Peavey, C., "Drag Prediction of Military Aircraft Using CFD," AIAA 2000-0383, 2000.

5. Cosner, R.R., "Assessment of Vehicle Performance Predictions Using CFD," AIAA 2000-0384, 2000.

6. Haase, W. et. al., "ECARP - European Aerodynamics Research Project: Validation of CFD Codes and Assessment of Turbulence Models," Notes on Numerical Fluid Mechanics, Vol 58, 1997

7. Redeker, G., "DLR-F4 Wing-Body Configuration," A Selection of Experimental Test Cases for the Validation of CFD Codes, AGARD Report AR-303, Aug. 1994.

8. Anon, "Proceedings of the First AIAA CFD Drag Prediction Workshop,"

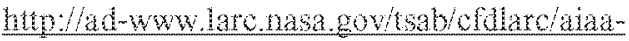
dpw/index.hml, and hup/wwwaiaa ore/tchaddragpredworkhop/Final Scholule and Resultshim, 2001.

9. Hemsch, M, "Statistical Analysis of CFD Solutions from the Drag Prediction Workshop," AIAA-2002$0842,2002$. 


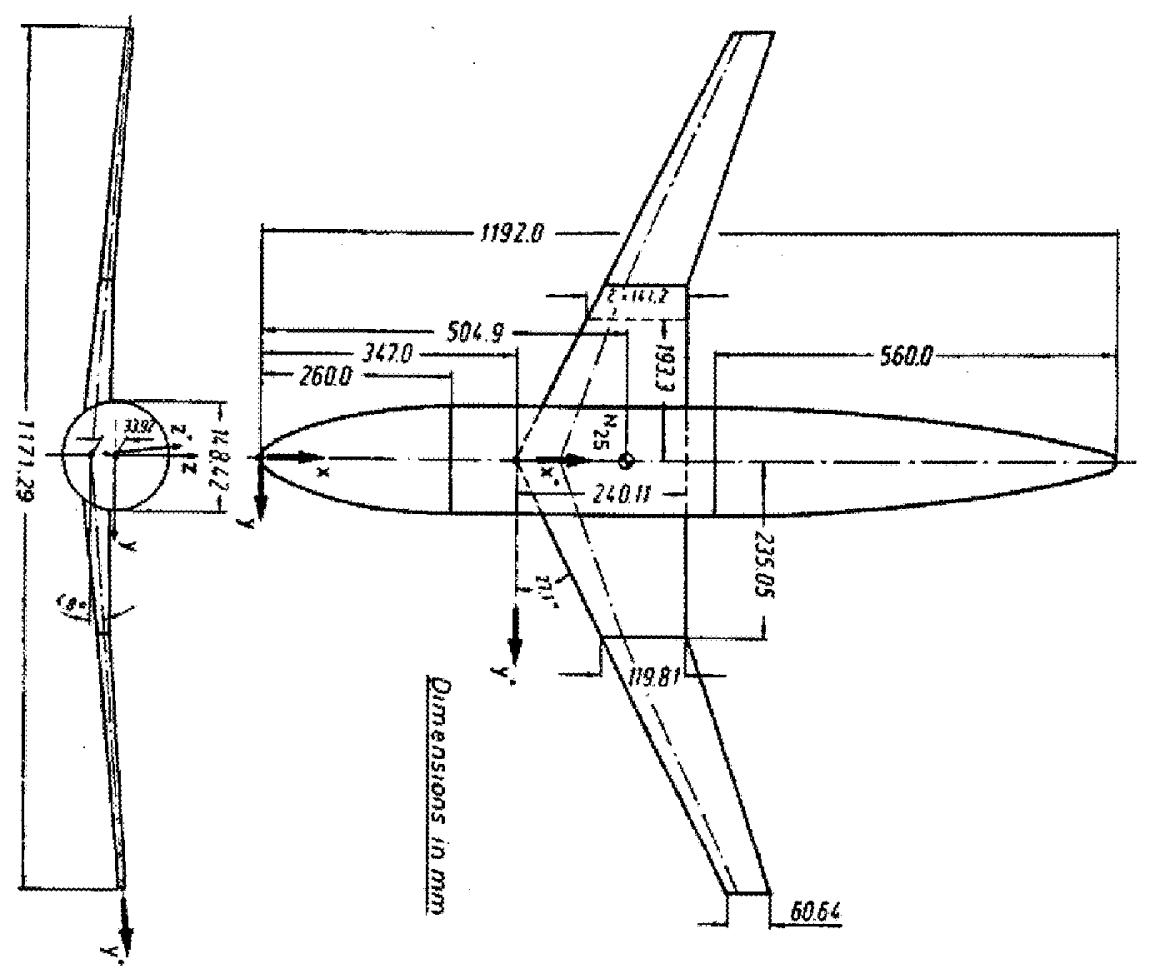

Figure 1. DLR-F4 Wing-Body Geometry (From Reference 7). 


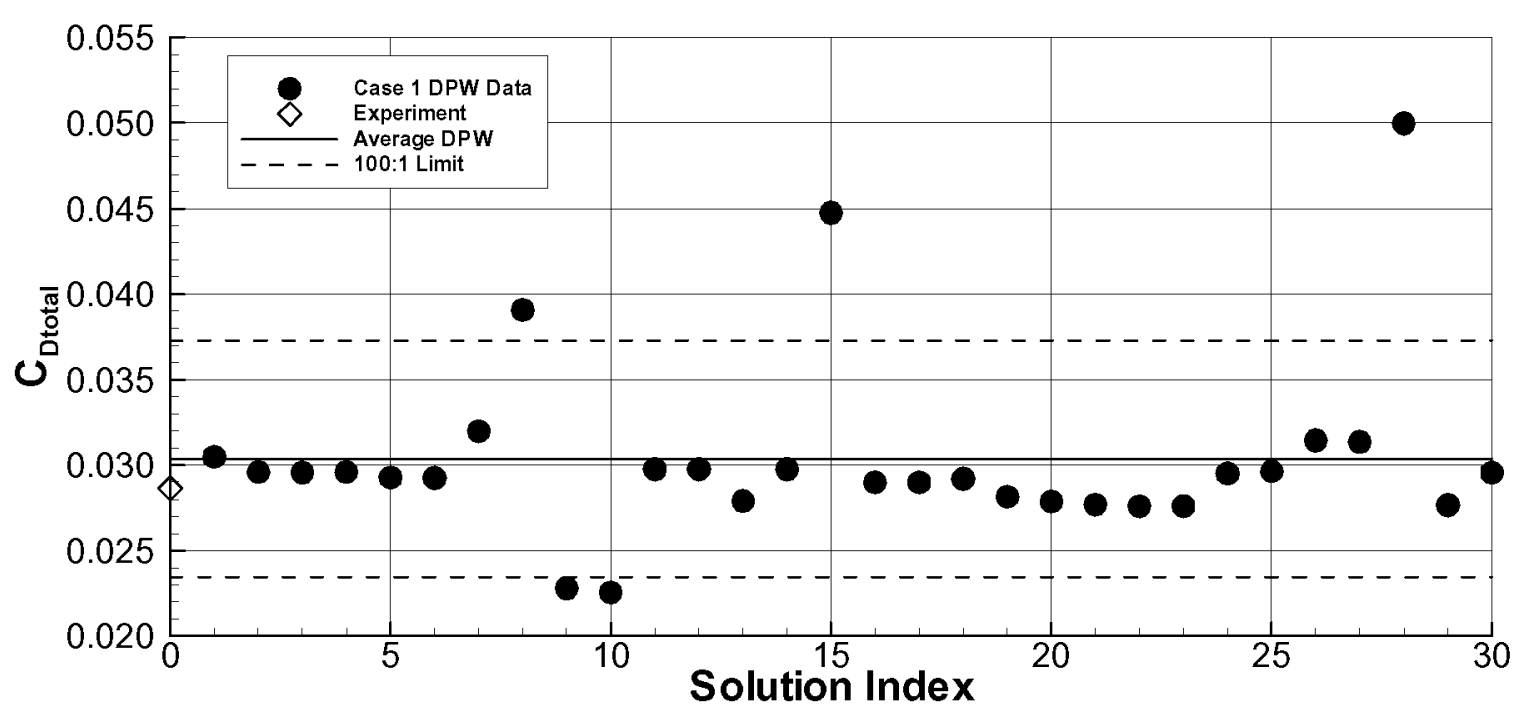

Figure 2. Total Drag Variations for Case 1: $M_{\infty}=0.75, C_{L}=0.500, R_{N_{c}}=3 \times 10^{6}$.

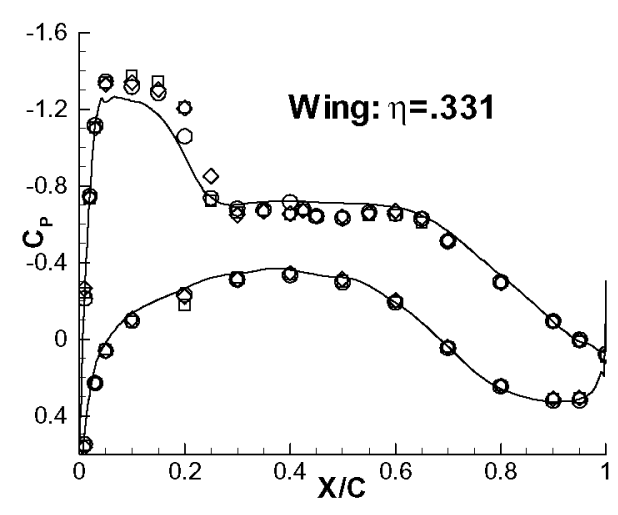

a) Typical Match

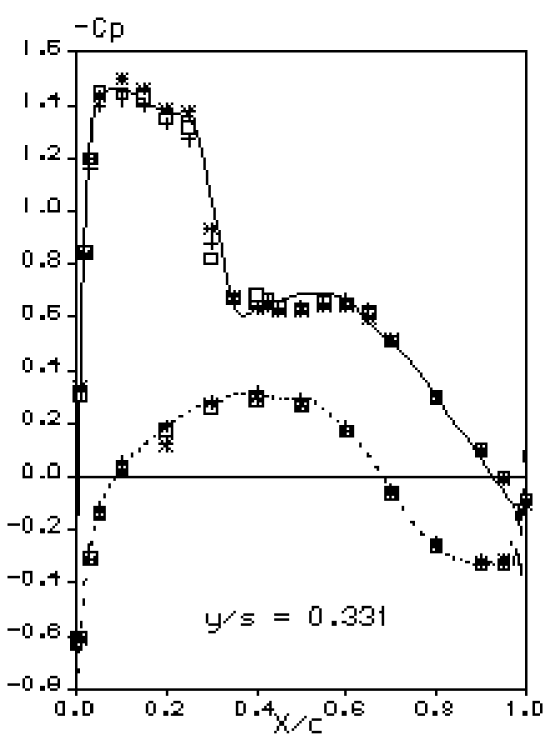

b) Better Match

Figure 3. Wing Pressure Profiles for Case 1: $M_{\infty}=0.75, C_{L}=0.500, R_{N c}=3 \times 10^{6}$. 
AIAA-2002-0841

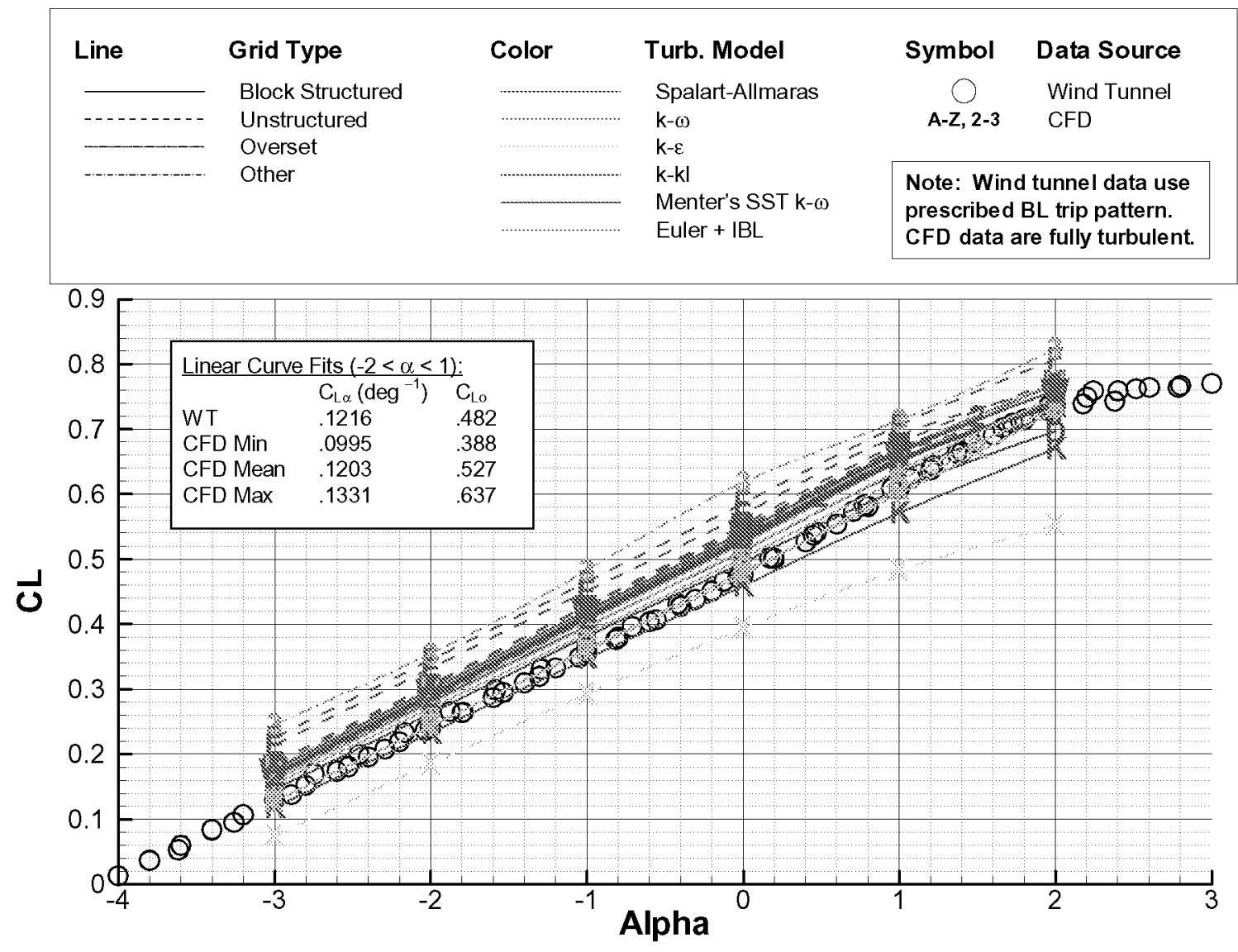

Figure 4. Composite Lift Curve Results for Case 2: $M_{\circ \circ}=0.75, R_{\mathrm{Nc}}=3 \times 10^{6}$.

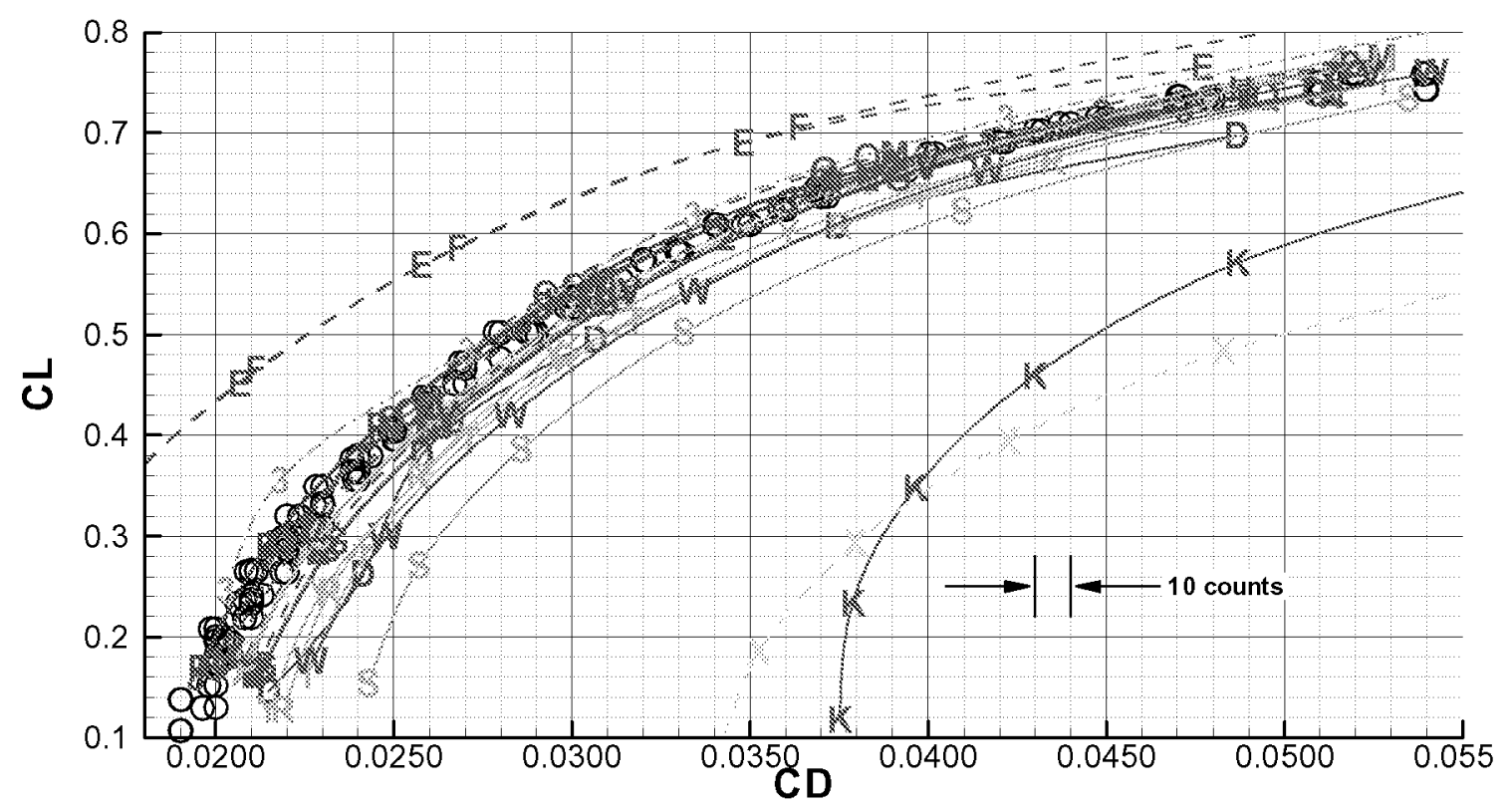

Figure 5. Composite Drag Polar Results for Case 2: $M_{\infty}=0.75, R_{\mathrm{Nc}}=3 \times 10^{6}$.

10

American Institute of Aeronautics and Astronautics 
AIAA-2002-0841

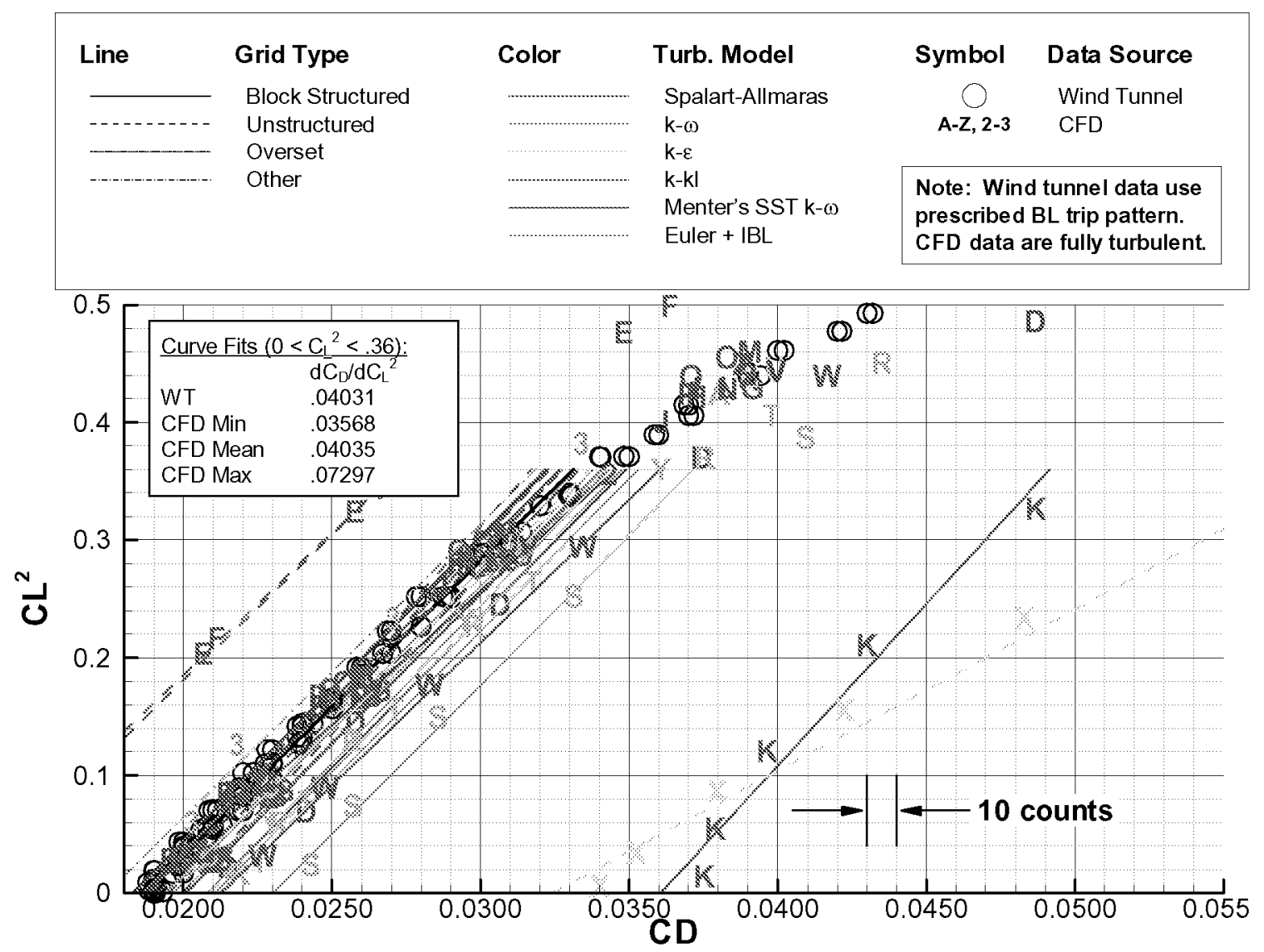

Figure 6. Composite Induced Drag Results for Case 2: $M_{\circ}=0.75, R_{\mathrm{Nc}}=3 \times 10^{6}$.

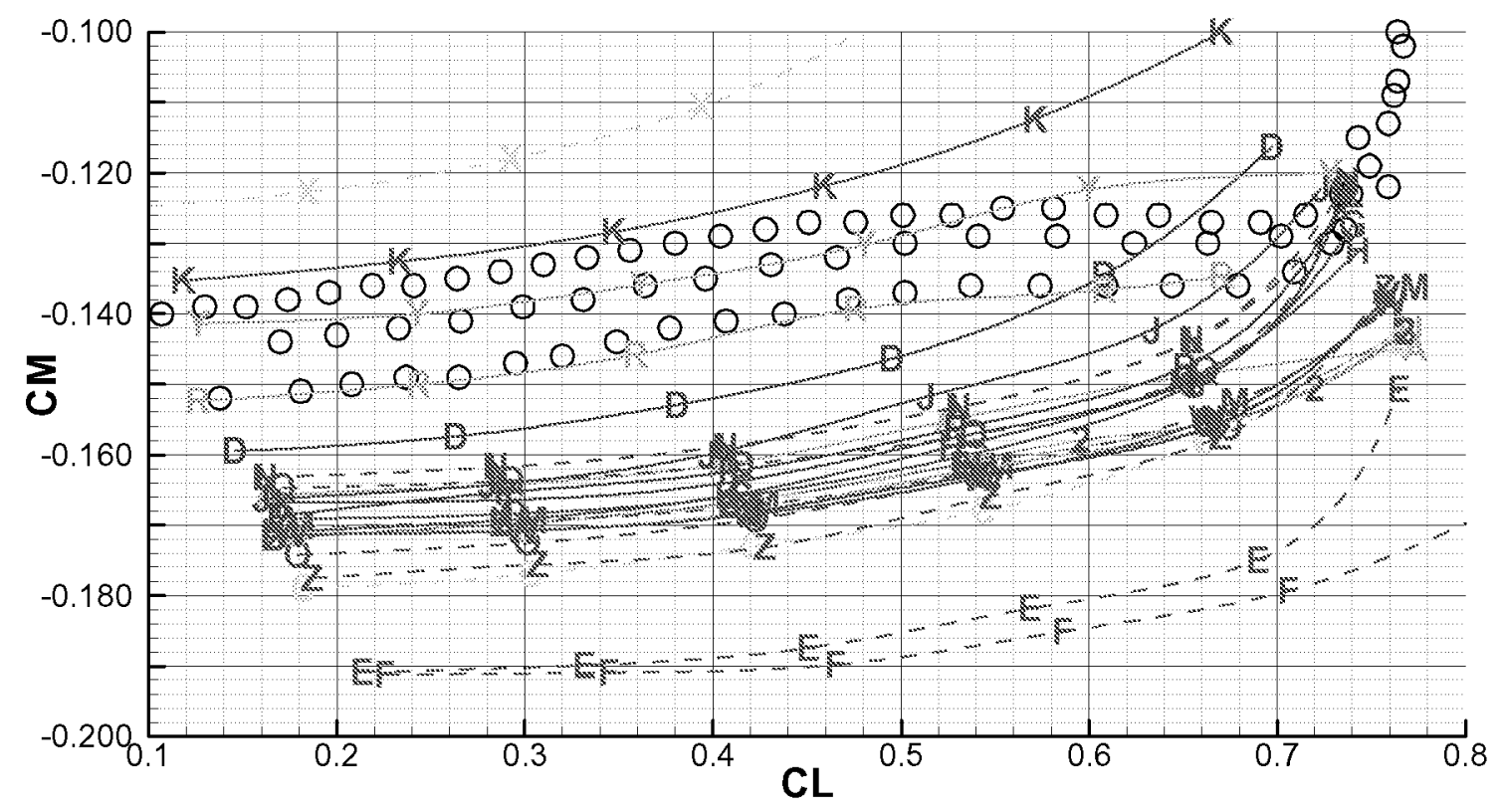

Figure 7. Composite Pitching Moment Results for Case 2: $M_{\infty}=0.75, R_{\mathrm{Nc}}=3 \times 10^{6}$. 

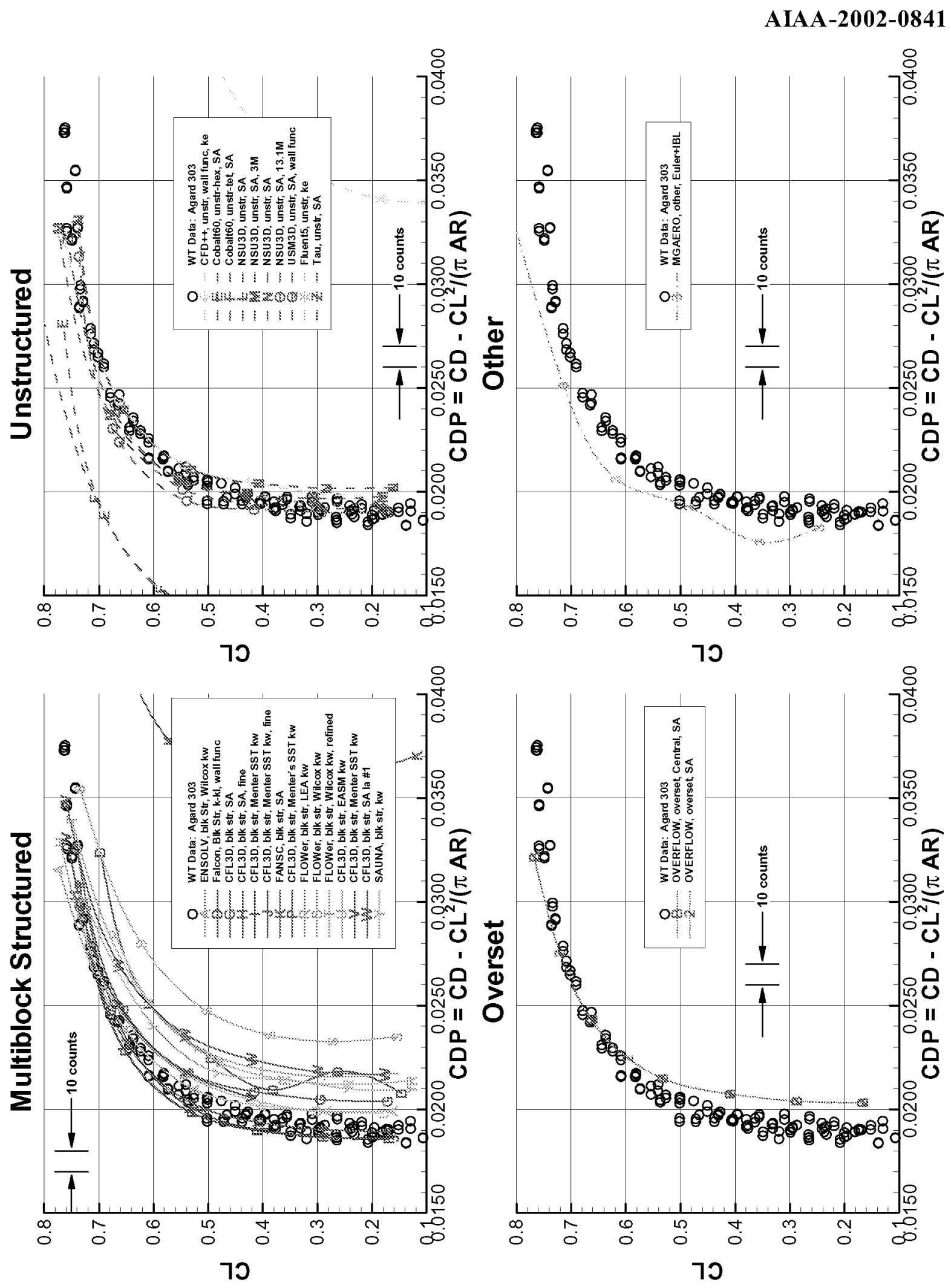

Figure 8. Trends with Grid Type for Case 2: $M_{\infty}=.75, R_{\mathrm{Nc}}=3 \times 10^{6}$. 
AIAA-2002-0841
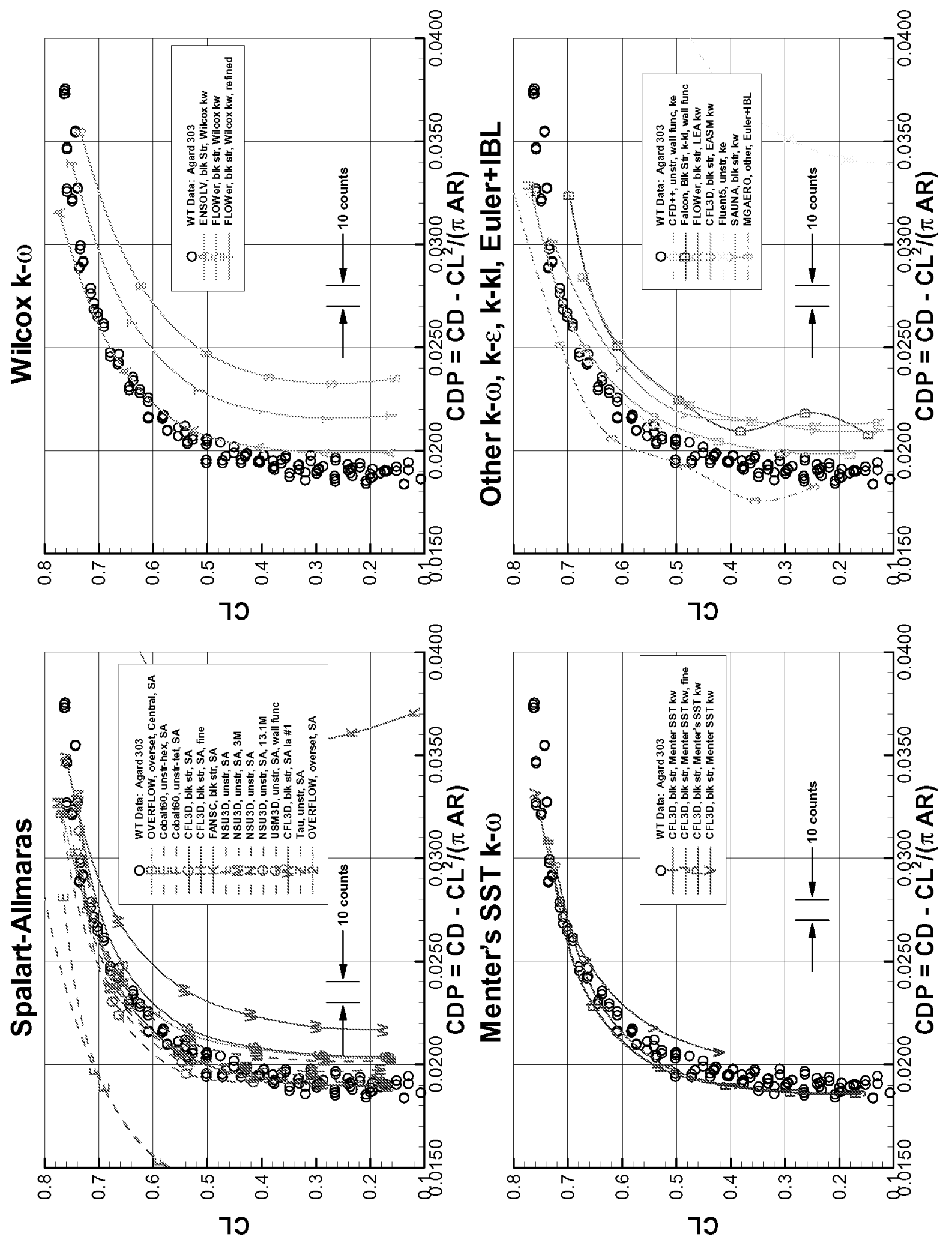

Figure 9. Trends with Turbulence Model for Case 2: $M_{\infty}=.75, R_{\mathrm{Nc}}=3 \times 10^{6}$. 
AIAA-2002-0841
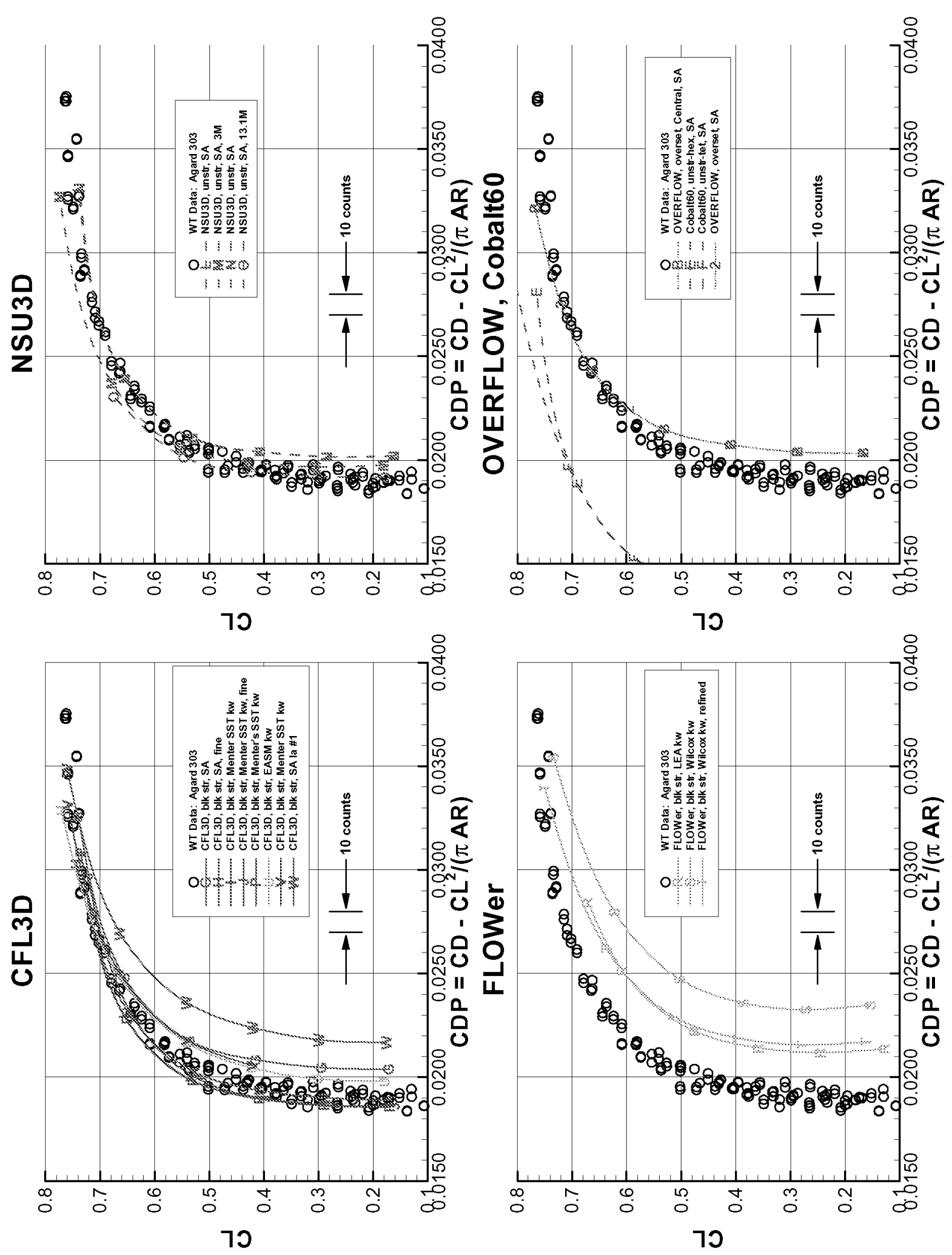

Figure 10. Trends by Code for Case 2: $M_{\infty}=.75, R_{\mathrm{Nc}}=3 \times 10^{6}$. 

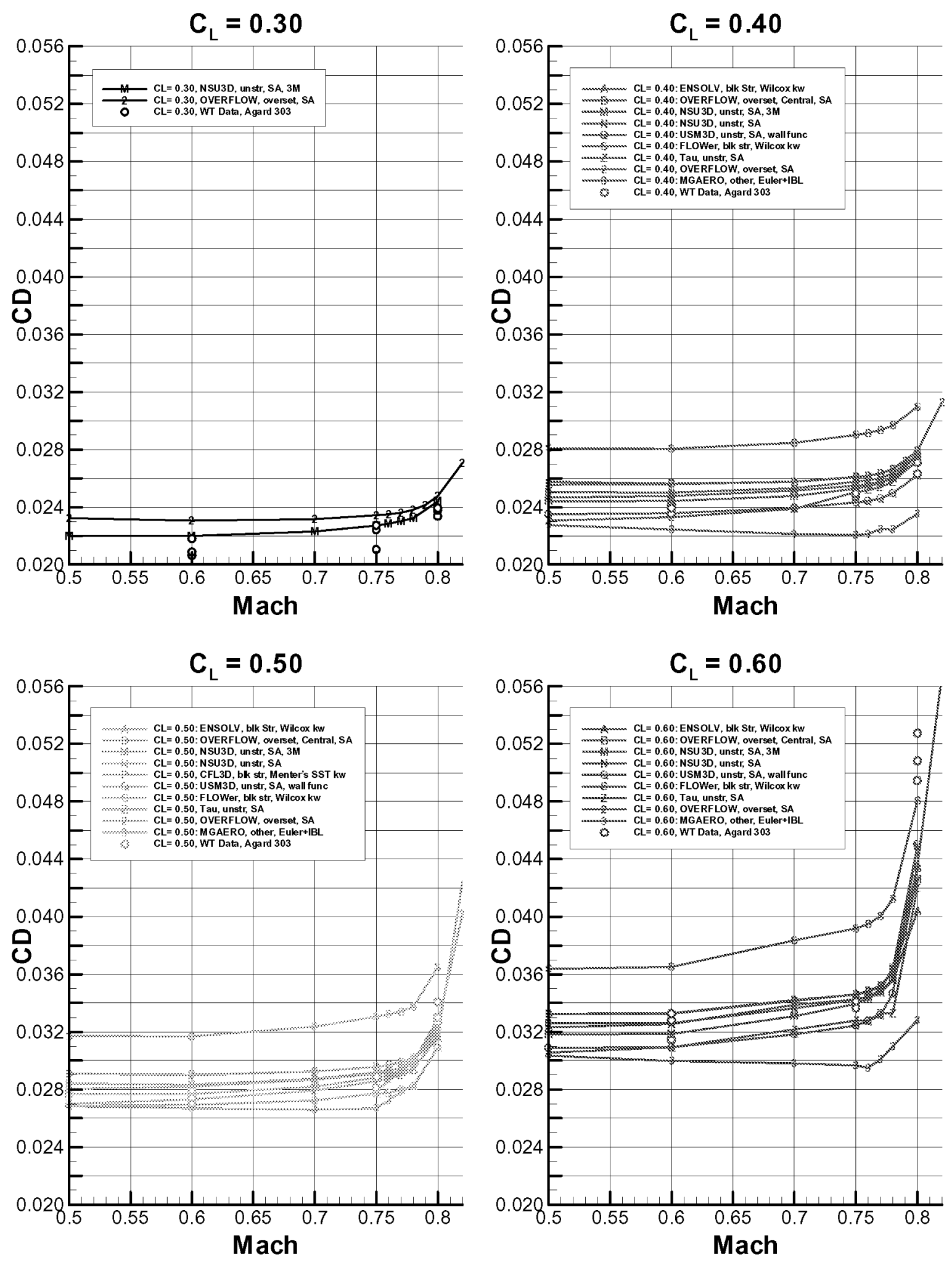

Figure 11. Drag Rise Results for Case 4: $R_{\mathrm{Nc}}=3 \times 10^{6}$.

American Institute of Aeronautics and Astronautics 